\title{
AN EXTENDED INEQUALITY FOR THE MAXIMAL FUNCTION
}

\author{
RICHARD J• BAGBY
}

ABSTRACT. Fefferman and Stein [3] have proved an $L^{p}$ inequality for the Hardy-Littlewood maximal function applied to functions taking values in a sequence space $l^{p}$. This note extends their theorem to functions taking values in a mixed $L^{p}$ space. An application to mixed estimates for Riesz potentials is given.

1. Notation. Let $k=\left(k_{1}, \cdots, k_{n}\right)$ be an ntuple of natural numbers, and let $P=\left(p_{1}, \cdots, p_{n}\right)$, where $1 \leq p_{i}<\infty$. For $f=\left\{f_{k}\right\}_{k \in N}, K_{P}(f)$ is defined by successively computing the $l^{p}$ norm with respect to $k_{i}$ while $k_{i+1}, \cdots$, $k_{n}$ are held fixed. The set of sequences on $N^{n}$ for which this norm is finite is denoted by $l^{P}$.

For $\phi$ a complex - valued locally integrable function on $R^{m}, \phi^{*}$ denotes the maximal function

$$
\phi^{*}(x)=\sup _{r>0} \frac{1}{m B(x, r)} \int_{B(x, r)}|\phi(y)| d y .
$$

Here $B(x, r)$ is the ball with center $x$ and radius $r ; m B(x, r)$ is its measure.

For $f=\left\{f_{k}\right\}$ a function on $R^{m}$ with values in $l^{P}, f^{*}$ is the $l^{P}$-valued function obtained by taking the maximal function of each $f_{k}$.

2. Theorem. For $1<p_{i}<\infty$ and $1<q<\infty$, there is a constant $c$ such that

$$
\int K_{P}\left(f^{*}\right)^{q} d x \leq c \int K_{P}(f)^{q} d x
$$

Proof. We perform induction on $n$. Fefferman and Ste in [3] have treated the case $n=1$; we could just as well start with $n=0$. Assume the Theorem has been established for some fixed value of $n$. We show that for $f=$ $\left\{f_{j, k}\right\}_{j \in N, k \in N^{n}}$ and $1<r<\infty$,

Received by the editors January 25, 1974.

AMS (MOS) subject classifications (1970). Primary 46E30, 26 A86.

Key words and phrases. Maximal function, mixed $L^{p}$ norm. 
420

R. J. BAGBY

$$
\int K_{P}\left[\left(\sum_{j=1}^{\infty}\left|f_{j, k}^{*}\right|^{r}\right)^{1 / r}\right]^{q} d x \leq c \int K_{P}\left[\left(\sum_{j=1}^{\infty}\left|f_{j, k}\right|^{r}\right)^{1 / r}\right]^{q} d x
$$

or, more briefly,

(*)

$$
\int K_{P} J_{r}\left(f^{*}\right)^{q} d x \leq c \int K_{P} J_{r}(f)^{q} d x .
$$

Note that for each $j, k$ we have

$$
\left|f_{j, k}(x)\right| \leq \sup _{j}\left|f_{j, k}(x)\right|=J_{\infty}\left(f_{\cdot, k}\right)(x) .
$$

Thus $f_{j, k}^{*}(x) \leq J_{\infty}\left(f_{\cdot, k}\right)^{*}(x)$, and so $J_{\infty}\left(f_{\cdot, k}^{*}\right) \leq J_{\infty}\left(f_{\cdot, k}\right)^{*}$. Hence

$$
\int K_{P} J_{\infty}\left(f^{*}\right)^{q} d x \leq \int K_{P}\left[J_{\infty}(f)^{*}\right]^{q} d x \leq c \int K_{P}\left[J_{\infty}(f)\right]^{q} d x
$$

by the inductive hypothesis. Thus (*) is valid for $r=\infty$.

Now suppose $1<r<\min \left(q, p_{1}, \cdots, p_{n}\right)$. Note that

$$
K_{P} J_{r}\left(f^{*}\right)=K_{P}\left[\left(\sum_{j=1}^{\infty}\left|f_{j,}^{*}\right|^{r}\right)^{1 / r}\right]=K_{P / r}\left[\sum_{j=1}^{\infty}\left|f_{j,}^{*} \cdot\right|^{r}\right]^{1 / r},
$$

where $P / r=\left(p_{1} / r, p_{2} / r, \cdots, p_{n} / r\right)$. Thus by the duality established in Renede and Canzone [2],

$$
\int K_{P} J_{r}\left(f^{*}\right)^{q} d x=\int K_{P / r}\left[\sum_{j=1}^{\infty}\left|f_{j,}^{*} \cdot\right|^{r}\right]^{q / r} d x=\sup _{\phi}\left|\int \sum_{k}\left[\sum_{j=1}^{\infty}\left|f_{j, k}^{*}\right|^{r}\right] \phi_{k} d x\right|^{q / r},
$$

where the supremum is taken over all $\phi=\left\{\phi_{k}\right\}$ for which $\int K_{(P / r)^{\prime}}(\phi)^{q /(q-r)} d x \leq 1$. By Lemma 1 of [3],

$$
\begin{aligned}
\left|\int_{k}\left[\sum_{j=1}^{\infty}\left|f_{j, k}^{*}\right|^{r}\right] \phi_{k} d x\right| \leq & \sum_{k} \sum_{j=1}^{\infty} \int\left|f_{j, k}^{*}\right|^{r}\left|\phi_{k}\right| d x \leq c \sum_{k} \sum_{j=1}^{\infty} \int\left|f_{j, k}\right|^{r} \phi_{k}^{*} d x \\
\leq & \int K_{P / r}\left[\sum_{j=1}^{\infty}\left|f_{j,}\right|^{r}\right] K_{(P / r)^{\prime}}\left[\phi^{*}\right] d x \\
\leq & \left(\int K_{P / r}\left[\sum_{j=1}^{\infty}\left|f_{j,}\right|^{r}\right]^{q / r} d x\right)^{r / q} \\
& \cdot\left(\int K_{\left.(P / r)^{,}\left[\phi^{*}\right]^{q /(q-r)} d x\right)^{1-r / q}}\right.
\end{aligned}
$$


using successive applications of Hölder's inequality for sequences and integrals. By the inductive hypothes is

$$
\int K_{(P / r)^{\prime}}\left[\phi^{*}\right]^{q /(q-r)} d x \leq c \int K_{(P / r)^{\prime}}[\phi]^{q /(q-r)} d x \leq c .
$$

Since $\int K_{P / r}\left[\Sigma_{j=1}^{\infty}\left|f_{j,} \cdot\right|^{r}\right]^{q / r} d x=\int K_{P} J_{r}(f)^{q} d x$, this yie lds (*).

We complete the proof by an interpolation process. Benedek and Panzone [2] give an extension of the Riesz-Thorin interpolation theorem to mixed $L^{p}$ spaces. However, the Theorem applies directly only to linear operators, and the operat or under consideration is nonlinear.

Instead we look at a linear operator $T$ defined by

$$
(T f)_{i, j, k}(x)=\frac{1}{m B\left(x, 2^{i}\right)} \int_{B\left(x, 2^{i}\right)} f_{j, k}(x) d x .
$$

Obviously

$$
I_{\infty}\left[(T f)_{\cdot, j, k}(x)\right]=\sup _{-\infty<i<\infty}\left|(T f)_{i, j, k}(x)\right| \leq f_{j, k}^{*}(x)
$$

while $f_{j, k}^{*}(x) \leq 2^{m} I_{\infty}\left[(T|f|)_{\cdot, j, k}(x)\right]$. (Here $\left.|f|=\left\{\left|f_{j, k}\right|\right\}_{.}\right)$Thus, (*) holds for all $f$ if and only if

$$
\int K_{P} J_{r} I_{\infty}(T f)^{q} d x \leq c \int K_{P} J_{r}(f)^{q} d x
$$

for all $f$.

We have seen that $(*)$ and, hence, $(* *)$ is valid for $r=\infty$ and for $1<r<$ $\min \left(q, p_{1}, \cdots, p_{n}\right)$; interpolation yields $(* *)$ for $1<r \leq \infty$. Thus, $(*)$ is valid for $1<r \leq \infty$ and the Theorem is proved.

3. A generalization. There is no particular difficulty in replacing the discrete variable $k$ by a continuous variable $t$. Let $\left(\Omega_{i}, \mu_{i}\right)$ be $\sigma$-finite measure spaces, and let $t=\left(t_{1}, \cdots, t_{n}\right) \in \Omega_{1} \times \Omega_{2} \times \cdots \times \Omega_{n}=\Omega$. For $f(x, t)$ a locally integrable function on $R^{m} \times \Omega$, let

$$
f^{*}(x, t)=\sup _{r>0} \frac{1}{m B(x, r)} \int_{B(x, r)}|f(y, t)| d y .
$$

Then we have $\int T_{P}\left(f^{*}\right)^{q} d x \leq c \int T_{P}(f)^{q} d x$, where $T_{P}$ denotes the mixed $L^{p}$ norm taken with respect to $t$.

4. An application. In [1], mixed norm estimates are obtained for Riesz potentials. One technique used there involves estimates in terms of a maximal function taken with respect to one of the variables. Then the proof is completed via the Calderón-Zygmund theory of singular integrals. $A_{n}$ 
alternate approach would be to use the Theorem stated above. This method would improve the exponent in Theorem 3 and remove the restriction $p_{l+1} \geq$ $p_{l+2} \geq \cdots \geq p_{n}$ in The orem $3^{\prime}$ of $[1]$.

\section{REFERENCES}

1. D. R. Adams and R. J. Bagby, Translation-dilation invariant estimates for Ries z potentials, Indiana Univ. Math. J. 23 (1974), 1051-1067.

2. A. Benedek and R. Panzone, The space $L^{p}$, with mixed norm, Duke Math. J. 28 (1961), 301-324. MR 23 \#A3451.

3. C. Fefferman and E. M. Stein, Some maximal inequalities, Amer. J. Math. 93 (1971), 107-115. MR 44 \#2026.

DE PARTMENT OF MATHEMATICS, NEW MEXICO STATE UNIVERSITY, LAS CRUCES, NEW MEXICO 88001

Current address: Mathematics Department, Washington University, St. Louis, Missouri 63130 